\title{
Types and applications of cough-related questionnaires
}

\author{
Zhijing Wang", Miao Wang", Siwan Wen, Li Yu, Xianghuai Xu \\ Department of Pulmonary and Critical Care Medicine, Tongji Hospital, Tongji University School of Medicine, Shanghai 200065, China \\ Contributions: (I) Conception and design: X Xu, L Yu; (II) Administrative support: None; (III) Provision of study materials or patients: None; (IV) \\ Collection and assembly of data: Z Wang, M Wang, S Wen; (V) Data analysis and interpretation: Z Wang, M Wang; (VI) Manuscript writing: All \\ authors; (VII) Final approval of the manuscript: All authors. \\ \#These authors contributed equally to this work. \\ Correspondence to: Xianghuai Xu; Li Yu. Department of Pulmonary and Critical Care Medicine, Tongji Hospital, Tongji University School of \\ Medicine, Shanghai 200065, China. Email: 05849@tongji.edu.cn; 96778@tongji.edu.cn.
}

\begin{abstract}
Cough is a normal protective reflex that can effectively remove foreign bodies and secretions from the airway. However, excessive cough adversely affects a patient's physiological, psychological and social functions. Thus, it is important to effectively evaluate the severity of cough to determine its diagnosis and treatment. Cough severity can be assessed with subjective or objective tools. Herein, we summarize the subjective evaluation of cough severity with questionnaires.
\end{abstract}

Keywords: Cough; questionnaire; measurement; quality of life (QOL)

Submitted Jun 12, 2019. Accepted for publication Sep 23, 2019.

doi: $10.21037 /$ jtd.2019.09.62

View this article at: http://dx.doi.org/10.21037/jtd.2019.09.62

\section{Introduction}

With the development of a biological-psychologicalsocial medical model, evaluating the influence of disease on physiology, psychology and society has been a focus in medical research. Studies have indicated that cough has a negative impact on a patient's physiological, psychological and social functions (1-3), and the adverse influence on the social-psychological domain is the most evident $(4,5)$. Successful treatment can not only relieve cough symptoms but also improve the quality of life (QOL) of these patients.

To accurately evaluate the health-related quality of life (HRQOL) of cough patients, investigators have designed a number of tools to assess cough severity and HRQOL: visual analogue scale (VAS), cough symptom score (CSS), Short Form-36 Health Survey (SF-36), St George's Respiratory Questionnaire (SGRQ), Leicester Cough Questionnaire (LCQ), Cough-specific Quality-oflife Questionnaire (CQLQ) and other respiratory health questionnaires (Table 1). These questionnaires are helpful for evaluating cough severity and therapeutic effects and for the clinical diagnosis and treatment of cough. Herein, we briefly introduced these questionnaires after classifying them into subjective cough score, HRQOL assessment and other cough-related questionnaires.

\section{Subjective cough score}

\section{Visual analogue scale (VAS)}

The VAS employs a linear scoring method that has a straight line with calibration of $0,1,2$ to $10 \mathrm{~cm}$ (scale lines marked from 0 to $100 \mathrm{~mm}$ can also be used); 0 indicates asymptomatic, and 10 represents the most serious (6). The patient is asked to mark the severity of cough on the line based on selfperception, and the distance between the starting point and the mark point is measured as the score. Studies have demonstrated the ability of a VAS to independently evaluate the symptoms and reflect the severity of the disease. The minimal important difference (MID) for the VAS has been reported to be $17 \mathrm{~mm}$ for acute cough (7), but the MID for chronic cough is still lacking published data.

In clinical practice, the VAS is widely used in the subjective evaluation and longitudinal assessment of cough, whether acute or chronic cough $(7,8)$. This scoring approach is easy and simple and less affected by language than other 
Table 1 Cough-related questionnaires

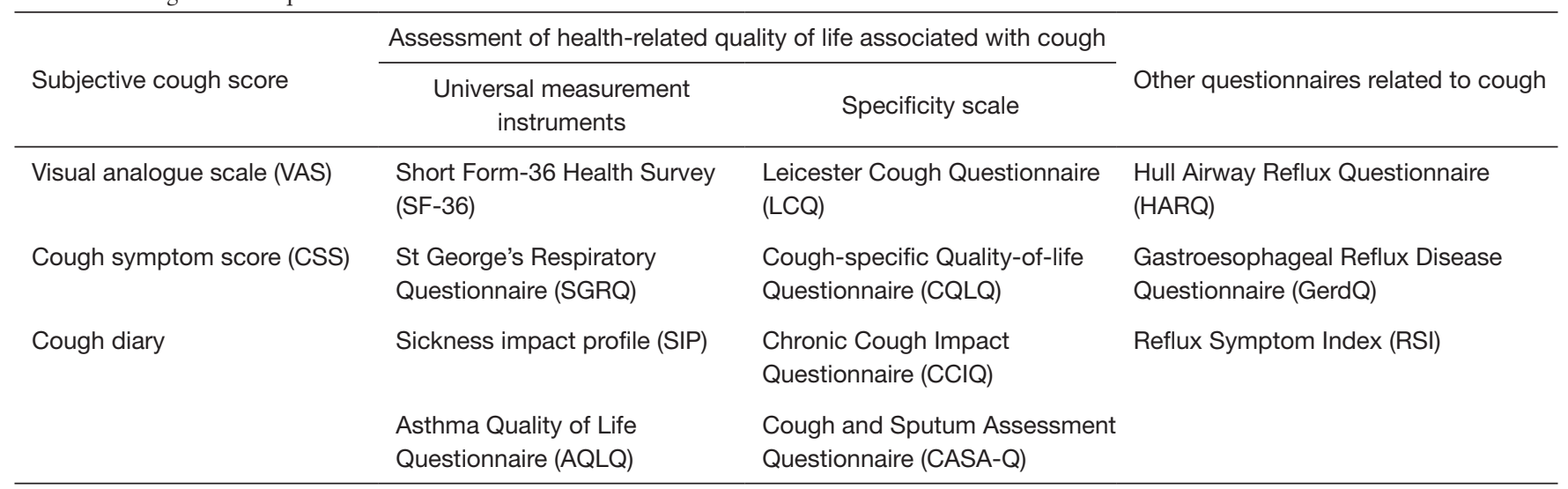

Table 2 Cough symptom score (CSS)

\begin{tabular}{lll}
\hline Score & Daytime & Night-time \\
\hline 0 & No cough during the day & No cough during the night \\
1 & Cough for one short period & Cough on waking only \\
2 & Cough for more than two short periods & Wake once or early due to cough \\
3 & Frequent coughing, which did not interfere with usual daytime & Frequent waking due to coughs \\
4 & activities & \\
5 & $\begin{array}{l}\text { Frequent coughing, which did interfere with usual daytime } \\
\text { activities }\end{array}$ & Frequent coughs most of the night \\
\hline
\end{tabular}

methods. Moreover, the VAS is highly responsive to changes in symptoms and is thus often used as an indicator in comparative studies of therapeutic effects $(6,9)$.

Although the role of the VAS has been recognized in both clinical and academic studies, the VAS also has certain limitations. It is strongly influenced by the subjective patient factors, and the evaluation may not be highly accurate.

\section{Cough symptom score (CSS)}

In the CSS (Table 2), the patient's intuitive perception and oral expression of cough are quantified. This score was first proposed by Hsu et al. in 1994, and its reliability and treatment response have been confirmed (10). The CSS is a two-part questionnaire referring to daytime and night-time symptoms. Based on the frequency, intensity and influence of cough on daily activities and sleep, cough symptoms are scored from 0 to 5 , with 0 indicating no cough and 5 indicating the most severe cough. In the study of the CSS, there was a significant correlation between the number of daytime cough incidents and the CSS in patients with chronic cough, but a weak correlation was noted between the CSS and night-time cough. This result indicates that the CSS can be used to evaluate the severity of daytime cough (10).

The simplified cough score (SCS) (Table 3) was recommended in the Guidelines for the Diagnosis and Treatment of Cough developed by the Respiratory Branch of Chinese Medical Association as a tool for evaluating the severity of cough. The SCS, which has evolved from the CSS, grades cough symptoms from 0 to 3 , which makes it simpler to use. We have proven the repeatability and response of the SCS to treatment, and it has a linear positive correlation with the CSS. Therefore, the SCS can also be used as an effective tool for the clinical assessment of cough severity along with the CSS (11). The abovementioned two scales are common in clinical work and studies, although the MID for them has not been researched. 
Table 3 Simplified cough score

\begin{tabular}{lll}
\hline Score & Daytime & Night-time \\
\hline 0 & No cough & No cough \\
1 & Transient cough occasionally during the daytime & Transient cough before sleep or occasional cough during the night \\
2 & Frequent cough mildly affecting daily life & Cough mildly affecting night sleep \\
3 & Frequent cough severely affecting daily life & Cough severely affecting night sleep \\
\hline
\end{tabular}

\section{Cough diary}

There is no concrete definition of a cough diary. Its application is very flexible and widely used in a large number of studies and clinical practice in different forms. This review introduces three main forms of cough diaries. Unfortunately, MID data are not available for any of these forms.

\section{Cough score record}

A cough score record is composed of subjective scores recorded every day over a period of time. With this method, cough can be rated with the CSS, the VAS or both, and the cough score is usually recorded independently after training. Children can score the cough under parental supervision, or their parents can record the scores based on the children's complaints. Post-treatment scores can be compared with pretreatment scores, and scores can also be compared with other measurements of cough severity. From the author's observation, cough score records have mainly been used in clinical research rather than clinical practice on account of the operational complexity of this method. Notably, the reliability and repeatability of cough score records have not been systematically confirmed.

\section{Diary card}

A patient diary card is a commonly used tool to record and evaluate cough symptoms, and information collected with a diary card is mainly employed for clinical research and investigations.

Gastroesophageal reflux-induced cough (GERC) is one of the causes of chronic cough, and the causality between reflux and cough is crucial for the diagnosis of GERC. Multichannel intraluminal impedance combined with $\mathrm{pH}$ monitoring (MII-pH) can be used to monitor reflux events in cough patients, but there is no effective measurement for the objective assessment of cough events. Therefore, investigators use cough diary cards to record the time and number of coughs and to calculate the symptom-related probability (SAP) or symptom index (SI) for the diagnosis of GERC $(12,13)$.

In some studies, cough diary cards are also used to selfassess cough symptoms, therapeutic efficacy and compliance with medication, and the results are recorded. The diary card is filled in by patients at night to recall medications and symptoms in the daytime. At the end of treatment, diary cards are retrieved to evaluate the therapeutic efficacy by assessing changes in the degree of cough symptoms during the treatment $(14,15)$.

\section{Cough severity diary (CSD)}

The cough severity diary (CSD), introduced by Vernon et al. (16) in 2010, is a simple tool for recording the scores of seven items that can effectively quantify cough severity. This tool can be used to investigate the progression of chronic and subacute cough and the efficacy of treatment. For a CSD, patients are asked to score their cough severity in three domains: cough frequency, cough intensity, and impact of cough on life and sleep. Patients recall their experience of cough within the last 24 hours and score their cough from 0 to 10 . The sum of CSD scores represents the severity of cough. The higher the score, the more serious the cough is.

Little is known about the application of this tool in cough patients, and it has been used in only a few studies. More studies with a large sample size are needed to confirm the validity and repeatability of this method.

\section{Assessment of HRQOL associated with cough}

\section{Universal measurement instruments}

Universal measurement instruments refer to questionnaires that can be widely used in the clinical assessment of HRQOL in patients with various diseases. These instruments have been widely applied in clinical settings with or without cough-related questions. 


\section{Short Form-36 Health Survey (SF-36)}

SF-36 is one of the most common instruments used to evaluate changes in health status in the prior year, and its reliability and effectiveness have been confirmed. SF-36 is composed of 8 multi-item scales (36 items) involving physical functioning $(\mathrm{PF})$, physical role functioning (RP), bodily pain (BP), general health $(\mathrm{GH})$, vitality (VT), social functioning (SF), role emotional (RE), and mental health (MH). Among them, PF, RF, BP and GH refer to physiological domains and constitute the Physical Component Summary (PCS). In contrast, RE, VT, MH and SF refer to the social-psychological domain and constitute the Mental Component Summary (MCS). In general, patients are asked to complete the questionnaire in less than $5 \mathrm{~min}$. The higher the score, the better the QOL is (17). The author found that no studies to date have examined the MID for SF-36 with respect to cough. SF-36 is not used for cough patients in clinical practice but may be very useful to compare QoL with non-coughing populations for research purpose.

For instance, in our clinical study, SF-36 scores were compared between chronic cough patients and healthy volunteers. The SF-36 scores of patients with chronic cough were significantly lower than those of healthy volunteers, and RE, GH and RP scores were significantly affected. In addition, compared to cough-specific scales such as LCQ, SF-36 can accurately reflect the health status of healthy volunteers without cough, which results in high comparability in HRQOL between cough and non-cough subjects (18).

It has been confirmed that SF-36 is effective for assessing QOL in patients with chronic cough, although the tool has poor performance in the postoperative assessment of cough in non-small cell lung cancer (NSCLC) patients. The investigators speculate that SF-36 is very complex and cannot be used to assess cough in NSCLC patients postoperatively (19).

\section{St George's Respiratory Questionnaire (SGRQ)}

SGRQ was introduced by Jones et al. in 1992 to measure the health of patients with chronic airflow obstruction (20). This questionnaire has 50 items involving 76 questions and includes the symptom (frequency and severity), activity (resulting in or affected by dyspnea), and disease impact (social function and psychological disorders caused by airway diseases) domains. The scores of each domain and total scores are weighted and range from 0 to 100 . The higher the score, the worse the HRQOL is. This disease- specific instrument assesses the impact of respiratory diseases on general health, daily life and perceived health, and it is meant to be completed independently by patients.

The SGRQ is mainly used in the clinical practice and research of patients with COPD and asthma, and it is also applied for the evaluation of HRQOL in idiopathic pulmonary fibrosis (IPF). Swigris et al. assessed the psychometric characteristics of the SGRQ in 1,061 patients with IPF treated with nintedanib or placebo (21). Their results support the use of the SGRQ to evaluate cough symptoms and HRQOL in IPF patients. This finding was consistent with the conclusions supported by the Australian IPF Registry (22) and the German IPF Registry (23). A change of 4 units in the SGRQ score is generally accepted in the literature to be the MID for the SGRQ in COPD (24); nevertheless, the MID for evaluating other diseases has not been reported.

\section{Sickness impact profile (SIP)}

The SIP is a universal health status measurement instrument designed to assess the functional status of patients with chronic disease. The reliability and validity of the tool have been confirmed in various studies. Although the clinical usefulness of the questionnaire is limited, researchers often use it for clinical researches.

The SIP consists of 136 items in 12 categories, and subjects are asked to complete the SIP questionnaire within $30 \mathrm{~min}$. Each item describes a specific behavioral disorder rather than a subjective self-assessment. Some categories belong to the physical level, while others belong to the social-psychological level; some independent categories are unrelated to both dimensions above. Only items that describe the dysfunction caused by the disease are completed. Each item is weighed after comparison with other items to reflect the relative severity of dysfunction (25). The MID for the SIP has not been studied.

Novitsky et al. used the SIP to investigate the HRQOL of patients with chronic cough caused by gastroesophageal reflux before and after anti-reflux surgery, and the results showed that the SIP was effective in evaluating the therapeutic efficacy of anti-reflux surgery (26) and thus deserves clinical applications.

\section{Asthma Quality of Life Questionnaire (AQLQ)}

The AQLQ, designed by Juniper et al. (27), consists of 32 items. It is divided into four domains: symptoms (12 items), activity restriction (11 items), affective function (5 items) and environmental stimulation (4 items). Each item has 
seven alternatives, with a score ranging from 1 to 7 and a high score indicating good QOL. A research suggests that the AQLQ has MID of 0.5 (28), and it is used to assess the HRQOL of asthma patients in the past two weeks. Subjects are asked to complete the AQLQ in 5-15 min. A number of studies have proven that the tool demonstrates favorable performance in the assessment of QOL and thus can be used to evaluate and identify QOL.

Riccioni et al. (29) used the AQLQ to assess the QOL of asthma patients, and the results showed that the tool was also effective in evaluating HRQOL in patients with simple cough symptoms. Notably, this tool applies only to clinical studies.

\section{Specificity scale}

\section{Leicester Cough Questionnaire (LCQ)}

In 2003, Birring et al. (30) designed the LCQ, which consisted of 19 items, including 8 physical items, 7 psychological items and 4 social items. Each item is rated according to the frequency of occurrence with a score ranging from 1 to 7 . The regional score is the sum of items scored divided by the items (score 1-7) in each area; the total score is the sum of the regional scores (3-21). The lower the score, the more serious the impact of chronic cough on QOL is. We have found that the MID for the LCQ in chronic cough is 1.3 (31). The LCQ is used not only in clinical work but also in some researches. In general, the LCQ is a brief, reproducible and accurate questionnaire for the assessment of chronic cough.

The Chinese version of the LCQ was first introduced in 2009 after achieving authorization from the investigators, and previous research has shown that the tool has high accuracy and repeatability (18). The score on the Chinese version of the LCQ for chronic cough patients is significantly higher than that for healthy controls, and the score decreases after treatment (12). The 2015 Cough Guideline recommends the Chinese version of the LCQ to assess the QOL of chronic cough patients (32). Ma et al. (18) and Shi et al. (33) found that there was no difference in LCQ scores among patients with chronic cough of different causes, which suggests that the LCQ is unable to identify the cause of chronic cough. The Department of Thoracic Surgery of West China Hospital of Sichuan University (34) employed a questionnaire to assess patients with thoracoscopic lung surgery, and the results showed that the LCQ in Mandarin-Chinese (LCQ-
MC) could be applied to evaluate cough after thoracoscopic surgery in patients with pulmonary diseases. Another group (35) investigated 129 patients with a simplified version of the LCQ-MC and examined its validity and reliability. Their results indicated that the simplified version of the LCQ-MC had good accuracy and reliability and could be used in the clinic. In addition to the Chinese version, the effectiveness of the Polish, Thai, Korean, and Dutch versions of the LCQ has also been confirmed in clinical studies (36-39).

\section{Cough-specific Quality-of-life Questionnaire (CQLQ)}

In 2002, French et al. improved their initial instrument, which contained 29 items and is a fixed-alternative, yes-orno questionnaire, and shortened it to 28 items. The score for each item was increased from $0-1$ to $1-4$, aiming to increase the sensitivity: 1 means strongly disagree; 2 means disagree; 3 means agree; and 4 means strongly agree. The questionnaire includes somatic symptoms, social psychology, functional ability, emotional state, extreme somatic symptoms and personal safety fears. The total score refers to the sum of the scores for 28 items, and the subscale score is the sum of the scores for each subscale item. The higher the score, the more serious the impact on QOL is (40). In general, clinical work, the questionnaire is rarely used to assess the effect of cough in patients, but it has been a useful tool in many clinical trials. The MID of the CQLQ is 13 for chronic cough (41). Studies have shown that the CQLQ is effective for the evaluation of both chronic and acute cough in adults and can be used to assess small changes in QOL with good reliability and accuracy (40). In addition, this questionnaire can be used for the objective evaluation of cystic fibrosis and COPD (42).

\section{Chronic Cough Impact Questionnaire (CCIQ)}

In 2005, Italian investigators (43) designed a new scale for a non-English-speaking population. The scale consists of 21 items, with 1-5 points for each item. It involves four domains reflecting the severity of cough in the last 2 weeks: daily activities, social activities, mood and sleep/attention. The higher the score, the larger the impact on QOL is. In patients with concomitant rhinitis, the Asthma Control Test (ACT) and the CCIQ are effective for managing asthma, especially for cough variant asthma (44). Currently, it is used in few clinical trials and is also used very little in clinical practice due to the language limitation. The MID for this tool has not been studied. 


\section{Cough and Sputum Assessment Questionnaire (CASA-Q)}

To evaluate cough and sputum symptoms and their impacts on daily activities in patients with COPD and chronic bronchitis, Crawford et al. developed and validated the CASA-Q (45). It is a self-report questionnaire based on the frequency, severity, and impact of cough and sputum during the prior 7 days. Lower scores indicate more severe symptoms or serious impacts. The questionnaire evaluates cough and sputum in two different ways-describing and evaluating the impact of two symptoms on QOL. In a global validation study of the CASA-Q, the results showed adequate internal consistency reliability and test-retest reliability and good correlations with partial correlated domains of SF-36 and SGRQ; however, the MID for the questionnaire was not mentioned. In addition, the scores for the cough symptom domain and sputum symptom domain of the CASA-Q are significantly responsive to deterioration or improvement in diseases, which is consistent with the results reported by Monz et al. (46).

These studies indicate that the CASA-Q may be used to assess cough and sputum symptoms and their impact in patients with COPD or chronic bronchitis. The main manifestations of airway mucus hypersecretion are chronic cough and expectoration, and the Chinese experts recommend that the CASA-Q be used to assess airway mucus hypersecretion in chronic airway inflammatory disease (47). In the author's opinion, this questionnaire is more suitable for clinical research and is too complex for the work of clinical staff.

\section{Other questionnaires related to cough}

\section{Hull Airway Reflux Questionnaire (HARQ)}

Morice et al. (48) designed the HARQ in 2011 to assess cough hypersensitivity. The questionnaire contains 14 items with $0-5$ points for each item and a total score of 70 points. The high scores suggest high cough hypersensitivity. It has been proven that the questionnaire is specific and accurate for evaluating airway reflux and cough hypersensitivity. In 2016, after authorization was obtained, we (49) translated the questionnaire into Chinese and confirmed that the Chinese version of the HARQ has good repeatability and may reflect responsiveness to treatment. The tool can effectively evaluate and distinguish chronic cough as well as its therapeutic efficacy; however, it cannot reflect the severity of cough. The favorable reliability and accuracy of the Swedish version of the HARQ have also been confirmed in clinical studies (50). The HARQ can be used to evaluate the therapeutic efficacy of ivacaftor in cystic fibrosis patients with cough (51), identify high airway reflux in most IPF patients (52), and predict the severity and frequency of acute episodes in non-cystic fibrosis bronchiectasis patients (53) In general, it is an available tool for clinical investigation $(51,54,55)$, but it may be a suboptimal choice for diagnosis and treatment. We did not find any survey examining the MID.

\section{Gastroesophageal Reflux Disease Questionnaire (GerdQ)}

The Reflux Disease Questionnaire (RDQ) is a symptombased medical history survey that has been proven effective and reliable in the diagnosis of gastroesophageal reflux disease (GERD) (56-58). However, due to its complexity, it is not widely used in clinical practice.

In 2007, Jones et al. (59) designed the GerdQ, which is a self-report and symptom-based tool for diagnosing GERD. The questionnaire is simplified from the RDQ and easy to understand and apply. It consists of 6 questions, including 4 positive reflux symptoms (reflux, heartburn, sleep disturbance caused by reflux and over-the-counter medication) and 2 negative reflux symptoms (upper abdominal pain and nausea). The MID for this tool has not been studied. A higher score indicates more serious symptoms (60). The questionnaire is widely applied in clinical performance and research on GERD and GERC. In our previous study, the anti-reflux effect of medications (61) was included as a diagnostic criterion for GERC, and the diagnostic value of GerdQ was further investigated in 126 patients with suspected GERC. Our results showed that when the GerdQ score was $\geq 8$, the diagnostic sensitivity was $67 \%$, specificity was $92 \%$, positive predicted value was $71 \%$, and negative predicted value was $96 \%$. Therefore, the GerdQ may be a useful indicator for the diagnosis of GERC, especially for GERC caused by acid reflux. However, the GerdQ is specifically designed for GERD and thus has limitations in the evaluation of cough symptoms. Moreover, some GERC patients have no acid reflux or heartburn. Therefore, we speculate that the GerdQ has limitations in the diagnosis of GERC and is not suitable for assessing GERC without reflux or heartburn.

\section{Reflux Symptom Index (RSI)}

Laryngopharyngeal reflux (LPR) refers to the reflux of 
stomach contents through the superior esophageal sphincter to the throat, respiratory tract and upper digestive tract. Currently, clinicians speculate that LPR is a serious form of gastroesophageal reflux and that omeprazole can achieve good efficacy in the treatment of LPR $(62,63)$. Belafsky et al. (64) designed the RSI and confirmed its reliability and validity in English. It has been accepted by increasing otolaryngologists and is widely used in many Englishspeaking countries (65). Some studies have utilized the scale in an Otolaryngology Department, a Pneumology Department and a Gastroenterology Department (66-68). However, whether it can be widely used in clinical work remains to be further studied. The MID is still unknown.

The Chinese version of the RSI is a relatively simple and feasible tool for the preliminary diagnosis and therapeutic evaluation of LPR with favorable reliability, validity and responsiveness, but more studies are needed to confirm the available findings (69).

\section{Conclusions}

In summary, there are many questionnaires for the evaluation of cough, and their clinical applications are quite different. Subjective cough scales are easy to use, and they have thus been widely used in clinical practice. However, they are easily affected by subjective factors such as emotion, attention to symptoms, and expectation. The assessment of HRQOL can comprehensively evaluate health-related QOL and its changes, but the questionnaires are complex, thus limiting its clinical use to some extent. The Chinese version of the LCQ is a valid tool for assessing coughrelated QOL, and its use and associated treatment response have been repeatedly studied in China. Other cough-related questionnaires are more flexible and have tangible value in the clinical evaluation and diagnosis of cough.

In clinical practice, the severity of cough cannot be evaluated solely with subjective methods such as questionnaires; objective methods are also needed to monitor cough and its response to treatment.

\section{Acknowledgments}

Funding: This study was supported by the National Natural Science Foundation of China (No. 81770097 and 81670092) and the Project of Science and Technology Commission of Shanghai Municipality (No. 17411970800).

\section{Footnotes}

Conflicts of Interest: The authors have no conflicts of interest to declare.

Ethical Statement: The authors are accountable for all aspects of the work in ensuring that questions related to the accuracy or integrity of any part of the work are appropriately investigated and resolved.

\section{References}

1. Birring SS, Spinou A. How best to measure cough clinically. Curr Opin Pharmacol 2015;22:37-40.

2. Chamberlain SA, Garrod R, Douiri A, et al. The impact of chronic cough: a cross-sectional European survey. Lung 2015;193:401-8.

3. Young EC, Smith JA. Quality of life in patients with chronic cough. Ther Adv Respir Dis 2010;4:49-55.

4. Dicpinigaitis PV, Tso R, Banauch G. Prevalence of depressive symptoms among patients with chronic cough. Chest 2006;130:1839-43.

5. Everett CF, Kastelik JA, Thompson RH, et al. Chronic persistent cough in the community: a questionnaire survey. Cough 2007;3:5.

6. Spinou A, Birring SS. An update on measurement and monitoring of cough: what are the important study endpoints? J Thorac Dis 2014;6:S728-34.

7. Lee KK, Matos S, Evans DH, et al. A longitudinal assessment of acute cough. Am J Respir Crit Care Med 2013;187:991-7.

8. Birring SS, Passant C, Patel RB, et al. Chronic tonsillar enlargement and cough: preliminary evidence of a novel and treatable cause of chronic cough. Eur Respir J 2004:23:199-201.

9. Wang K, Birring SS, Taylor K, et al. Montelukast for postinfectious cough in adults: a double-blind randomised placebo-controlled trial. Lancet Respir Med 2014;2:35-43.

10. Hsu JY, Stone RA, Logan-Sinclair RB, et al. Coughing frequency in patients with persistent cough: assessment using a 24 hour ambulatory recorder. Eur Respir J 1994;7:1246-53.

11. Lai K. Chinese National Guidelines on Diagnosis and Management of Cough: consensus and controversy. J Thorac Dis 2014;6:S683-8.

12. Dong R, Xu X, Yu L, et al. Randomised clinical trial: gabapentin vs baclofen in the treatment of suspected 
refractory gastro-oesophageal reflux-induced chronic cough. Aliment Pharmacol Ther 2019;49:714-22.

13. Yang Z, Xu X, Chen Q, et al. The diagnostic value of symptom index in gastroesophageal reflux-induced chronic cough. Zhonghua Nei Ke Za Zhi 2014;53:108-11.

14. Birring SS, Brew J, Kilbourn A, et al. Rococo study: a realworld evaluation of an over-the-counter medicine in acute cough (a multicentre, randomised, controlled study). BMJ Open 2017;7:e014112.

15. Nandha D, Goodyer L, Woodruffe-Peacock C. Diary cards and the assessment of cough symptoms in community pharmacies. Pharm World Sci 2000;22:17-20.

16. Vernon M, Kline Leidy N, Nacson A, et al. Measuring cough severity: development and pilot testing of a new seven-item cough severity patient-reported outcome measure. Ther Adv Respir Dis 2010;4:199-208.

17. Lins L, Carvalho FM. SF-36 total score as a single measure of health-related quality of life: Scoping review. SAGE Open Med 2016;4:2050312116671725.

18. Ma W, Yu L, Wang Y, et al. Changes in health-related quality of life and clinical implications in Chinese patients with chronic cough. Cough 2009;5:7.

19. Lin R, Che G. Validation of the Mandarin Chinese version of the Leicester Cough Questionnaire in non-small cell lung cancer patients after surgery. Thorac Cancer 2018;9:486-90.

20. Jones PW, Quirk FH, Baveystock CM, et al. A selfcomplete measure of health status for chronic airflow limitation. The St. George's Respiratory Questionnaire. Am Rev Respir Dis 1992;145:1321-7.

21. Swigris JJ, Wilson H, Esser D, et al. Psychometric properties of the St George's Respiratory Questionnaire in patients with idiopathic pulmonary fibrosis: insights from the INPULSIS trials. BMJ Open Respir Res 2018;5:e000278.

22. Glaspole IN, Chapman SA, Cooper WA, et al. Healthrelated quality of life in idiopathic pulmonary fibrosis: Data from the Australian IPF Registry. Respirology 2017;22:950-6.

23. Kreuter M, Swigris J, Pittrow D, et al. Health related quality of life in patients with idiopathic pulmonary fibrosis in clinical practice: insights-IPF registry. Respir Res 2017;18:139.

24. Jones PW. St. George's Respiratory Questionnaire: MCID. COPD 2005;2:75-9.

25. Ton TG, Watson NF, Koepsell TD, et al. Narcolepsy and the Sickness Impact Profile: A general health status measure. Sleep Sci 2014;7:5-12.
26. Novitsky YW, Zawacki JK, Irwin RS, et al. Chronic cough due to gastroesophageal reflux disease: efficacy of antireflux surgery. Surg Endosc 2002;16:567-71.

27. Juniper EF, Guyatt GH, Epstein RS, et al. Evaluation of impairment of health related quality of life in asthma: development of a questionnaire for use in clinical trials. Thorax 1992;47:76-83.

28. Juniper EF, Guyatt GH, Willan A, et al. Determining a minimal important change in a disease-specific Quality of Life Questionnaire. J Clin Epidemiol 1994;47:81-7.

29. Riccioni G, D'Orazio N, Di Ilio C, et al. Quality of Life and clinical symptoms in asthmatic subjects. J Asthma 2004;41:85-9.

30. Birring SS, Prudon B, Carr AJ, et al. Development of a symptom specific health status measure for patients with chronic cough: Leicester Cough Questionnaire (LCQ). Thorax 2003;58:339-43.

31. Raj AA, Pavord DI, Birring SS. Clinical cough IV:what is the minimal important difference for the Leicester Cough Questionnaire? Handb Exp Pharmacol 2009:311-20.

32. Society CT. Diagnostic and Treatment Guideline of Cough (2015). Chin J Tubercul Respir Dis 2016;39:323-54.

33. Shi X, Peng QF, Kong LF. An analysis of factors for quality-of-life in patients with chronic cough. Zhonghua Nei Ke Za Zhi 2011;50:672-5.

34. Xu Z, Lin R, Che G, et al. Validation of the Mandarin Chinese Version of the Leicester Cough Questionnaire in Patients Undergoing Lung Resection for Patients with Lung Disease. Zhongguo Fei Ai Za Zhi 2017;20:389-94.

35. Lin R, Che G, Xu Z, et al. Simplification and Validation of Leicester Cough Questionnaire in Mandarin-Chinese. Zhongguo Fei Ai Za Zhi 2017;20:468-72.

36. Dabrowska M, Krakowiak K, Radlinska O, et al. Validation of the Polish Version of the Chronic Cough Quality of Life Questionnaire (Leicester Cough Questionnaire). Adv Clin Exp Med 2016;25:649-53.

37. Huisman AN, Wu MZ, Uil SM, et al. Reliability and validity of a Dutch version of the Leicester Cough Questionnaire. Cough 2007;3:3.

38. Kwon JW, Moon JY, Kim SH, et al. Reliability and validity of a korean version of the leicester cough questionnaire. Allergy Asthma Immunol Res 2015;7:230-3.

39. Pornsuriyasak P, Kawamatawong T, Rattanasiri S, et al. Validity and reliability of the Thai version of the leicester cough questionnaire in chronic cough. Asian Pac J Allergy Immunol 2016;34:212-6.

40. French CT, Irwin RS, Fletcher KE, et al. Evaluation 
of a cough-specific quality-of-life questionnaire. Chest 2002;121:1123-31.

41. Fletcher KE, French CT, Irwin RS, et al. A prospective global measure, the Punum Ladder, provides more valid assessments of quality of life than a retrospective transition measure. J Clin Epidemiol 2010;63:1123-31.

42. Smith J, Owen E, Earis J, et al. Cough in COPD: correlation of objective monitoring with cough challenge and subjective assessments. Chest 2006;130:379-85.

43. Baiardini I, Braido F, Fassio O, et al. A new tool to assess and monitor the burden of chronic cough on quality of life: Chronic Cough Impact Questionnaire. Allergy 2005;60:482-8.

44. Braido F, Baiardini I, Balestracci S, et al. The relationship between asthma control and quality-of-life impairment due to chronic cough: a real-life study. Ann Allergy Asthma Immunol 2008;101:370-4.

45. Crawford B, Monz B, Hohlfeld J, et al. Development and validation of a cough and sputum assessment questionnaire. Respir Med 2008;102:1545-55.

46. Monz BU, Sachs P, McDonald J, et al. Responsiveness of the cough and sputum assessment questionnaire in exacerbations of COPD and chronic bronchitis. Respir Med 2010;104:534-41.

47. Shen Y, Huang S, Kang J, et al. Management of airway mucus hypersecretion in chronic airway inflammatory disease: Chinese expert consensus (English edition). Int J Chron Obstruct Pulmon Dis 2018;13:399-407.

48. Morice AH, Faruqi S, Wright CE, et al. Cough hypersensitivity syndrome: a distinct clinical entity. Lung 2011;189:73-9.

49. Huang Y, Yu L, Xu XH, et al. Validation of the Chinese version of Hull airway reflux questionnaire and its application in the evaluation of chronic cough. Zhonghua Jie He He Hu Xi Za Zhi 2016;39:355-61.

50. Johansson EL, Ternesten-Hasséus E. Reliability and Validity of the Swedish Version of the Hull Airway Reflux Questionnaire (HARQ-S). Lung 2016;194:997-1005.

51. Faruqi S, Shiferaw D, Morice AH. Effect of Ivacaftor on Objective and Subjective Measures of Cough in Patients with Cystic Fibrosis. Open Respir Med J 2016;10:105-8.

52. Fahim A, Dettmar PW, Morice AH, et al. Gastroesophageal reflux and idiopathic pulmonary fibrosis: a prospective study. Medicina (Kaunas) 2011;47:200-5.

53. Mandal P, Morice AH, Chalmers JD, et al. Symptoms of airway reflux predict exacerbations and quality of life in bronchiectasis. Respir Med 2013;107:1008-13.

54. Smith JE, Morjaria JB, Morice AH. Dietary intervention in the treatment of patients with cough and symptoms suggestive of airways reflux as determined by Hull airways Reflux Questionnaire. Cough 2013;9:27.

55. Faruqi S, Wilmot R, Wright C, et al. Serum LDH in chronic cough: a potential marker of airway inflammation. Clin Respir J 2012;6:81-7.

56. Aanen MC, Numans ME, Weusten BL, et al. Diagnostic value of the Reflux Disease Questionnaire in general practice. Digestion 2006;74:162-8.

57. Nocon M, Kulig M, Leodolter A, et al. Validation of the Reflux Disease Questionnaire for a German population. Eur J Gastroenterol Hepatol 2005;17:229-33.

58. Shaw M, Dent J, Beebe T, et al. The Reflux Disease Questionnaire: a measure for assessment of treatment response in clinical trials. Health Qual Life Outcomes 2008;6:31.

59. Jones R, Junghard O, Dent J, et al. Development of the GerdQ, a tool for the diagnosis and management of gastro-oesophageal reflux disease in primary care. Aliment Pharmacol Ther 2009;30:1030-8.

60. Lacy BE, Chehade R, Crowell MD. A prospective study to compare a symptom-based reflux disease questionnaire to 48 -h wireless $\mathrm{pH}$ monitoring for the identification of gastroesophageal reflux (revised 2-26-11). Am J Gastroenterol 2011;106:1604-11.

61. Xu X, Chen Q, Liang S, et al. Comparison of gastroesophageal reflux disease questionnaire and multichannel intraluminal impedance $\mathrm{pH}$ monitoring in identifying patients with chronic cough responsive to antireflux therapy. Chest 2014;145:1264-70.

62. Johnston N, Dettmar PW, Strugala V, et al. Laryngopharyngeal reflux and GERD. Ann N Y Acad Sci 2013;1300:71-9.

63. Kowalik K, Krzeski A. The role of pepsin in the laryngopharyngeal reflux. Otolaryngol Pol 2017;71:7-13.

64. Belafsky PC, Postma GN, Amin MR, et al. Symptoms and findings of laryngopharyngeal reflux. Ear Nose Throat J 2002;81:10-3.

65. Belafsky PC, Postma GN, Koufman JA. Validity and reliability of the reflux symptom index (RSI). J Voice 2002;16:274-7.

66. Habermann W, Schmid C, Neumann K, et al. Reflux symptom index and reflux finding score in otolaryngologic practice. J Voice 2012;26:e123-7.

67. Jiang N, Sung CK, Damrose EJ. Improvement in the Reflux Symptom Index Following Surgery for Cricopharyngeal Dysfunction. J Voice 2017;31:86-9.

68. Kaplan M, Tanoglu A, Erkul E, et al. Association of reflux 
symptom index scores with gastroesophageal flap valve status. Auris Nasus Larynx 2014;41:543-7.

69. Zheng JY, Zhang LH, Li JJ, et al. Chinese version of the

Cite this article as: Wang $\mathrm{Z}$, Wang $\mathrm{M}$, Wen $\mathrm{S}$, Yu $\mathrm{L}, \mathrm{Xu}$

$\mathrm{X}$. Types and applications of cough-related questionnaires. J

Thorac Dis 2019;11(10):4379-4388. doi: 10.21037/jtd.2019.09.62 reflux symptom index was evaluated for reliability and validity. Zhonghua Er Bi Yan Hou Tou Jing Wai Ke Za Zhi 2012;47:894-8. 